\title{
Consistent estimation of real NMP zeros in stable LTI systems of arbitrary complexity
}

\author{
Cristian R. Rojas* Håkan Hjalmarsson* László Gerencsér ** \\ Jonas Mårtensson* \\ * ACCESS Linnaeus Center, Electrical Engineering, \\ KTH-Royal Institute of Technology, S-100 44 Stockholm, Sweden \\ ** Computer and Automation Institute of the Hungarian Academy of \\ Sciences, (MTA SZTAKI), POB 63, H-1518 Budapest, Hungary
}

\begin{abstract}
In this contribution we show that under certain conditions it is possible to estimate a non-minimum phase zero consistently using a very simple 2 parameter finite impulse response model, for arbitrarily complex finite dimensional stable linear time invariant systems.
\end{abstract}

\section{INTRODUCTION}

How to cope with system complexity is a key issue in system identification. A model is always an idealization of the real world, yet the purpose of modeling is to capture characteristics of the real system behaviour that are important for the application at hand, despite that the complete system behavior cannot be modelled. That meaningful modeling should be based on the intended model use has long been recognized, see for example [Wahlberg and Ljung, 1986, Gevers and Ljung, 1986, Ljung, 1992]. This issue was very much brought into focus during the efforts, initiated in the early 1990s, to address the so called identification for control problem [Gevers, 1991, Schrama, 1992, Goodwin et al., 1992, Gevers, 1993, Bayard et al., 1992, Jacobsen, 1994, Zang et al., 1995, Van den Hof and Schrama, 1995, de Callafon and Van den Hof, 1997, Böling and Mäkilä, 1998, Goodwin, 1999, Rivera and Jun, 2000, Malan et al., 2001, Eker and Nikolaou, 2002].

A key outcome here was the importance of the experiment design $^{1}$ and this lead to iterative approaches trying to achieve experimental conditions such that the bias error was suitably distributed over frequencies to suit control applications.

However, also computational methods for optimal experiment design were revisited and extended [Lindqvist and Hjalmarsson, 2000, 2001, Hildebrand and Gevers, 2003, Jansson and Hjalmarsson, 2004, 2005].

In [Barenthin et al., 2005] it is illustrated that optimal input design may result in significant savings in experimental efforts in control applications. Through some simple examples, it was advocated in [Hjalmarsson, 2005] that it is possible to combat the curse of complexity, i.e. that the model uncertainty grows with the system complexity so that for highly complex systems the model becomes virtually useless, by careful experiment design and that this also allows simple models to be used (as long as only a limited amount of system properties are to be extracted from the measurements). The cost of an experiment vs the amount of information to be extracted has been formalized in [Rojas et al., 2008a,b] for frequency function estimation.

\footnotetext{
* This work was supported in part by the Swedish Research Council under contract

1 This, of course, was well known earlier on also but during this time its importance became very palpable.
}

Following up on [Hjalmarsson, 2005], the dual role of a "good" input as 1) an enhancer of system properties of interest, and 2) as an attenuator of properties of little or no interest was formalized in [Hjalmarsson et al., 2006] and further developed in Mårtensson [2007]. In particular it was shown that, under certain conditions, an input that is designed to be optimal for a scalar cost function and for a full order model, results in experimental data for which also reduced order models can be used to consistently identify the property of interest.

The special case of identification of non-minimum phase system zeros had previously been considered in [Jansson, 2004, Mårtensson et al., 2005] where it was shown that the input

$$
u_{t}=z_{o}^{-1} u_{t-1}+r_{t-1}
$$

where $z_{o}$ is the NMP-zero of interest and $\left\{r_{t}\right\}$ is zero mean white noise, allows $z_{o}$ to be consistently identified using a two parameter FIR model. Even though conceptually interesting, the catch of this result from a practical point of view is, of course, that the input depends on the zero to be identified. Hence, even though it is shown in [Jansson, 2004] that the choice of the pole of the input spectrum is not so critical for the accuracy of the estimate, the practical applicability of the result is limited. It is common that an optimal experiment design depends on the true system and there are two main approaches to circumvent this problem: 1) robust input designs where the design takes into account that the true system is uncertain a priori Rojas [2008], and 2) adaptive, or sequential, designs where the design is successively updated as new data is collected from the system [Lindqvist and Hjalmarsson, 2002, Gerencsér and Hjalmarsson, 2005]. Recently, it has been shown [Gerencsér et al., 2007, 2009] that when the true system is in the model set and when an ARX model is used, adaptive input design achieves asymptotically (in the sample size) the same accuracy as a non-adaptive design where the true system is known. See also the recent survey [Pronzato, 2008].

In this contribution we re-visit the zero estimation problem and propose an adaptive approach. We consider stable causal discrete-time linear time-invariant systems with real valued zeros outside the unit circle, subject to stationary stochastic disturbances. We show that it is possible to estimate the right-most of these zeros consistently using a simple two parameter FIR model, if the input can be manipulated. The main contribution is the convergence 
analysis of this algorithm. We remark that existing convergence results of recursive identification/adaptive control algorithms in the case of severe under-modeling, which is the case in this contribution, are very limited in the literature.

\section{PROBLEM DEFINITION}

Consider the system where

$$
y_{t}=G_{o}(\mathrm{q}) u_{t}+v_{t}^{o}
$$

where $G_{o}(\mathrm{q})$ is a stable rational transfer function, where $u_{t}$ and $y_{t}$ represent the measured input and output, respectively, where $v_{t}^{o}=H_{o}(q) e_{t}^{o}$ for some stable and inversely stable rational transfer function $H_{o}(\mathrm{q})$ and with $\left\{e^{o}\right\}$ being a sequence of independent (but not necessarily identically distributed) random variables of zero mean, variance $\lambda_{o}$ and bounded moments of every order.

The system $G_{o}$ has a real-valued NMP-zero at an unknown location $z_{o}$ which we would like to estimate using the very simple model

$$
y_{t}=\varphi_{t}^{T} \theta
$$

where $\varphi_{t}=\left[\begin{array}{ll}u_{t-1} & u_{t-2}\end{array}\right]^{T}$.

In [Mårtensson, 2007] it is shown that if the input is chosen as

$$
u_{t}=\frac{c}{1-z_{o}^{-1} \mathrm{q}^{-1}} r_{t}
$$

where $r_{t}$ is zero mean white noise with unit variance, then prediction error identification using the model (2) will, as the sample size grows, converge to a model which has $z_{o}$ as zero! We will call this signal a " $z_{o}$-consistent input". Notice that for quite general model structures, when the true system is in the model set, this input is actually optimal for estimating $z_{o}$ [Mårtensson et al., 2005].

A simple proof when the system itself is of FIR type, but possibly subject to colored noise, and is operating in open loop, is as follows. Let the true system be described by

$$
y_{t}=\tilde{\varphi}_{t}^{T} \theta^{o}+v_{t}^{o}
$$

where $\theta^{o} \in \mathbb{R}^{n_{o}}, v_{t}=H_{o}(\mathrm{q}) e_{t}^{o}$ and $\tilde{\varphi}_{t}=\left[u_{t-1} \ldots u_{t-n_{o}}\right]$. Extend $\theta$ to

$$
\theta^{e}=\left[\begin{array}{c}
\theta \\
0_{\left(n_{o}-2\right) \times 1}
\end{array}\right] \in \mathbb{R}^{n_{o}}
$$

Then, assuming open loop operation, the normal equations when the sample size $N \rightarrow \infty$ can be written

$$
\mathbf{E} \varphi_{t} \tilde{\varphi}_{t}^{T}\left(\theta^{o}-\theta^{e}\right)=0
$$

Now with $\Gamma(z)=\left[\begin{array}{lll}z^{-1} & \ldots z^{-n_{o}}\end{array}\right], \Gamma^{T}\left(z_{o}\right) \theta^{o}=0$ defines a zero $z_{o}$ of the system. The objective is now to show that the limit model should have the same zero, i.e. $\Gamma^{T}\left(z_{o}\right) \theta^{e}=0$, i.e. that we have the relationship

$$
\left.\begin{array}{c}
0=\Gamma^{T}\left(z_{o}\right)\left(\theta^{o}-\theta^{e}\right) \\
z_{o} \text { zero of the system }
\end{array}\right\} \Rightarrow z_{o} \text { zero of the model }
$$

Now the first row in (3) is given by

$$
\left[r_{u}(0) r_{u}(1) \ldots r_{u}\left(n_{o}-1\right)\right]\left(\theta^{o}-\theta^{e}\right)=0
$$

and, hence, if we choose the input such that its correlation function satisfies

$$
r_{u}(k)=c \cdot z_{o}^{-k}, \quad k=0,1, \ldots, n_{o}
$$

for some arbitrary $c>0$, then (5) can be written

$$
c z_{o} \Gamma^{T}\left(z_{o}\right)\left(\theta^{o}-\theta^{e}\right)=0
$$

Thus an input with correlation function (6) will, by (4), imply that $z^{o}$ is a zero of the limit model.
Now, unfortunately, $z_{o}$ is the quantity to be estimated and thus the choice (6) is not possible. However, suppose that we have an initial guess (or estimate) $\hat{z}_{o}$ of the NMPzero location $z_{o}$. Following [Gerencsér and Hjalmarsson, 2005, Gerencsér et al., 2007, 2009], a way to cicumvent the problem would then be to use an adaptive (or sequential) approach where recursive identification is combined with a time-varying input filter in which, referring to the certainty equivalence principle, the unknown zero $z_{0}$ is replaced by the latest available estimate of this zero.

\section{THE ALGORITHM}

The algorithm we will analyse is a very simple modification of the RLS algorithm for the two parameter model (2), together with the adaptive input

$$
u_{t}=\rho_{t-1} u_{t-1}+\sqrt{1-\rho_{t-1}^{2}} r_{t-1}
$$

where $\rho_{t}$ will depend on the parameter estimate, and $\left\{r_{t}\right\}$ is a sequence of independent (but not necessarily identically distributed) random variables of zero mean, unit variance and bounded moments of every order, which is independent of $\left\{e_{t}^{o}\right\}$. The motivation for generating the input as in (7) is that should $\rho_{t-1}$ happen to be equal to $z_{o}^{-1}$, then, according to the result in the previous section, the RLS-estimate for the model (2) corresponds to a consistent estimate of $z_{o}$. We are thus trying to use the input to bootstrap the parameter estimate to the desired value. The objective of the next section is to analyse when this may work but first let us present the details of the algorithm.

The RLS-algorithm for the model (2) is given by

$$
\begin{aligned}
\hat{\theta}(t) & =\hat{\theta}(t-1)+\frac{1}{t} R_{t}^{-1} \varphi_{t}\left(y_{t}-\hat{y}_{t}\right) \\
R_{t} & =R_{t-1}+\frac{1}{t}\left(\varphi_{t} \varphi_{t}^{T}-R_{t-1}\right)
\end{aligned}
$$

Note that, if $\rho_{t}=\rho$ in $(7)$, then $R_{t}$ will converge to

$$
R(\rho):=\left[\begin{array}{ll}
1 & \rho \\
\rho & 1
\end{array}\right]
$$

and based on this we suggest to replace $R_{t}$ in (8) by $R\left(\rho_{t-1}\right)$, resulting in the algorithm

$$
\hat{\theta}(t)=\hat{\theta}(t-1)+\frac{1}{t} R^{-1}\left(\rho_{t-1}\right) \varphi_{t}\left[y_{t}-\hat{y}_{t}\right]
$$

Notice that this algorithm is extremely simple; it can be written as

$$
\begin{aligned}
\hat{\theta}_{1}(t) & =\hat{\theta}_{1}(t-1)+\frac{1}{t\left(1-\rho_{t-1}^{2}\right)}\left[u_{t-1}-\rho_{t-1} u_{t-2}\right]\left[y_{t}-\hat{y}_{t}\right] \\
\hat{\theta}_{2}(t) & =\hat{\theta}_{2}(t-1)+\frac{1}{t\left(1-\rho_{t-1}^{2}\right)}\left[u_{t-2}-\rho_{t-1} u_{t-1}\right]\left[y_{t}-\hat{y}_{t}\right] \\
\hat{y}_{t} & =\hat{\theta}_{1}(t-1) u_{t-1}+\hat{\theta}_{2}(t-1) u_{t-2}
\end{aligned}
$$

The zero for this simple model is given by

$$
\hat{z}_{t}=-\frac{\hat{\theta}_{2}(t)}{\hat{\theta}_{1}(t)}
$$

and we will thus use

$$
\rho_{t}=\frac{1}{\hat{z}_{t}}=-\frac{\hat{\theta}_{1}(t)}{\hat{\theta}_{2}(t)}
$$

in $(7)$.

Summarizing, the proposed algorithm is given by 


$$
\begin{aligned}
\hat{\theta}(t) & =\hat{\theta}(t-1)+\frac{1}{t} R^{-1}\left(\rho_{t-1}\right) \varphi(t)\left[y_{t}-\hat{y}_{t}\right] \\
u_{t} & =-\frac{\hat{\theta}_{1}(t)}{\hat{\theta}_{2}(t)} u_{t-1}+\sqrt{1-\frac{\hat{\theta}_{1}^{2}(t)}{\hat{\theta}_{2}^{2}(t)}} r_{t-1}
\end{aligned}
$$

Notice that the algorithm performs a division by $\hat{\theta}_{2}^{2}(t)$. In order to avoid a possible division by zero, a projection mechanism, such as the one described in (17), can be applied. Another reason for using such a technique is to ensure convergence, as explained in the next section.

\section{CONVERGENCE ANALYSIS}

We will now provide a formal convergence analysis of (9)(10) when the true system is given by (1), where $G_{o}$ is a stable rational transfer function with exactly one pure time delay, i.e.

$$
G_{o}(q)=\sum_{k=1}^{\infty} g_{k} q^{-k}, \quad g_{1} \neq 0
$$

and with at least one real NMP zero, and where $H_{o}$ is stable. We will employ the ODE analysis in [Ljung, 1977] so we begin by considering the "frozen" system, i.e. the system when $\hat{\theta}(t)=\theta$ and $\rho_{t}=\rho$ are fixed. This system is given by

$$
\begin{aligned}
u_{t}(\rho) & =-\rho u_{t-1}(\rho)+\sqrt{1-\rho^{2}} r_{t-1} \\
y_{t}(\rho) & =G_{o}(q) u_{t}(\rho)+e_{t} \\
\varphi_{t}(\rho) & =\left[\begin{array}{l}
u_{t-1}(\rho) \\
u_{t-2}(\rho)
\end{array}\right] \\
\hat{y}_{t}(\theta, \rho) & =\varphi_{t}^{T}(\rho) \theta \\
\rho & =\rho(\theta):=-\frac{\theta_{1}}{\theta_{2}}
\end{aligned}
$$

The associated ODE is given by

$$
\dot{\theta}=f(\theta, \rho(\theta))
$$

where

$$
f(\theta, \rho)=R^{-1}(\rho) \mathbf{E}\left[\varphi_{t}(\rho)\left(y_{t}(\rho)-\hat{y}_{t}(\theta, \rho)\right)\right]
$$

Notice that since $\left\{e_{t}^{o}\right\}$ is independent of $\left\{r_{t}\right\}$, and since

$$
\mathbf{E}\left[u_{t}(\rho) u_{t-k}(\rho)\right]=\rho^{|k|},
$$

it holds that

$$
\begin{aligned}
\mathbf{E}\left[u_{t-l}(\rho) y_{t}(\rho)\right] & =\mathbf{E}\left[u_{t-l}(\rho) G_{o}(q) u_{t}(\rho)\right] \\
& =\sum_{k=1}^{\infty} g_{k} \mathbf{E}\left[u_{t-l}(\rho) u_{t-k}(\rho)\right] \\
& =\sum_{k=1}^{\infty} g_{k} \rho^{|k-l|}
\end{aligned}
$$

and hence

$$
\begin{aligned}
& R^{-1}(\rho) \mathbf{E}[\varphi(t, \rho) y(t, \rho)] \\
& =\frac{1}{1-\rho^{2}}\left[\begin{array}{cc}
1 & -\rho \\
-\rho & 1
\end{array}\right]\left[\begin{array}{c}
g_{1}+\sum_{k=2}^{\infty} \rho^{k-1} g_{k} \\
\rho g_{1}+\sum_{k=2}^{\infty} \rho^{k-2} g_{k}
\end{array}\right] \\
& =\frac{1}{1-\rho^{2}}\left[\begin{array}{c}
-\sum_{k=2}^{\infty} \rho^{k} g_{k}+\sum_{k=2}^{\infty} \rho^{k-2} g_{k}
\end{array}\right] \\
& =\left[\begin{array}{c}
g_{1} \\
\rho^{-2} \sum_{k=2}^{\infty} \rho^{k} g_{k}
\end{array}\right] \\
& =\left[\begin{array}{c}
\rho^{-2} G_{o}\left(\rho^{-1}\right)-g_{1} \rho^{-1}
\end{array}\right]
\end{aligned}
$$

Inserting (13) and

$$
R^{-1}(\rho) \mathbf{E}\left[\varphi_{t}(\rho) \hat{y}_{t}(\theta, \rho)\right]=\theta
$$

in (12) gives

$$
f(\theta, \rho)=\left[\begin{array}{c}
g_{1}-\theta_{1} \\
\rho^{-2} G_{o}\left(\rho^{-1}\right)-g_{1} \rho^{-1}-\theta_{2}
\end{array}\right]
$$

so that

$$
f(\theta, \rho(\theta))=\left[\left(\frac{\theta_{1}}{\theta_{2}}\right)^{-2} G_{o}\left(-\frac{g_{1}-\theta_{1}}{\theta_{1}}\right)+\frac{\theta_{2}}{\theta_{1}}\left(g_{1}-\theta_{1}\right)\right]
$$

Let us now introduce the change of variables $z=-\theta_{2} / \theta_{1}$, i.e. we use the zero as one of the variables. We have

$$
\begin{aligned}
\dot{z} & =-\frac{\dot{\theta}_{2}}{\theta_{1}}+\frac{\theta_{2}}{\theta_{1}^{2}} \dot{\theta}_{1} \\
& =-\frac{z \dot{\theta}_{1}+\dot{\theta}_{2}}{\theta_{1}} \\
& =-\frac{z\left(g_{1}-\theta_{1}\right)+z^{2} G_{o}(z)-z\left(g_{1}-\theta_{1}\right)}{\theta_{1}} \\
& =-\frac{z^{2} G_{o}(z)}{\theta_{1}}
\end{aligned}
$$

Thus (11) corresponds to the following ODE

$$
\begin{aligned}
\dot{\theta}_{1} & =g_{1}-\theta_{1} \\
\dot{z} & =-\frac{z^{2} G_{o}(z)}{\theta_{1}}
\end{aligned}
$$

To analyze the stability of this ODE, notice that (14) is globally asymptotically stable at $\theta_{1}=g_{1}$, and for (15) we can define the function

$$
V(z):=\frac{1}{2}\left(z-z_{o}\right)^{2}
$$

where $z_{o}$ is a real NMP zero of $G_{o}$. Notice that $V$ is positive definite (i.e., $V(z) \geq 0$ for all $z \in \mathbb{R}$, and $V(z)=0$ if and only if $z=z_{o}$ ), and

$$
\dot{V}(z)=-\frac{z^{2} G_{o}(z)}{\theta_{1}}\left(z-z_{o}\right)
$$

For large $t$, we have that $\theta_{1}$ has the same sign as $g_{1}$. From (16) and the facts that $\lim _{z \rightarrow+\infty} z G(z)=g_{1}$ and $G(z)$ only changes sign at its real zeros, we see that $\dot{V}<0$ if and only if there is an even number of (or no) real zeros greater than $z_{o}$. This is the condition for the local stability of $z_{o}$, whose domain of attraction (in case it is stable) is the interval $[a, b]$, where $a$ and $b$ are the zeros of $G$ immediately to the left and right of $z_{o}$, respectively. 
We can now refer to Corollary 1 in [Ljung, 1977] (with the set of assumptions B from that paper) to conclude that for the algorithm (9)-(10) equipped with a projection mechanism which restricts the trajectory of $z_{t}$ to lie in a closed set $D_{z} \subset(-\infty,-1) \cup(1, \infty)$ which is strictly inside the domain of attraction of a given real zero $z_{0}$, and the trajectory of $\hat{\theta}_{1}$ to lie in a compact set $D_{\theta_{1}}$, it holds that

$$
\begin{aligned}
\hat{\theta}_{1}(t) \rightarrow \theta_{1}^{o} & \text { w.p. } 1 \\
\hat{z}(t) \rightarrow z_{o} & \text { w.p. } 1
\end{aligned}
$$

or that the estimation sequence has a cluster point on the boundary of the projection area. The derivation parallells the proof of Lemma 7.A.1 in [Ljung and Söderström, 1983].

As an example of such a projection mechanism, we can replace (9) by

$$
\hat{\theta}(t)=\left\{\hat{\theta}(t-1)+\frac{1}{t} R^{-1}\left(\rho_{t-1}\right) \varphi(t)\left[y_{t}-\hat{y}_{t}\right]\right\}_{D}
$$

where

$$
\left\{\left[\begin{array}{l}
\theta_{1} \\
\theta_{2}
\end{array}\right]\right\}_{D}:=\left\{\begin{array}{l}
{\left[\begin{array}{l}
\theta_{1} \\
\theta_{2}
\end{array}\right], \text { if } \theta_{1} \in D_{\theta_{1}} \text { and }-\theta_{2} / \theta_{1} \in D_{z}} \\
{\left[\begin{array}{l}
\theta_{1}^{o} \\
\theta_{2}^{o}
\end{array}\right], \text { otherwise }}
\end{array}\right.
$$

with $\theta_{1}^{o}$ and $\theta_{2}^{o}$ are such that $\theta_{1}^{o}$ and $-\theta_{2}^{o} / \theta_{1}^{o}$ lie in the interior of $D_{\theta_{1}}$ and $D_{z}$, respectively. See [Ljung and Söderström, 1983] for more details.

\section{NUMERICAL EXAMPLE}

In this section we illustrate the use of the algorithm presented in Section 3 with a simple example, which exhibits the performance of the algorithm compared to the use of the $z_{o}$-consistent input for the estimation of the NMP zero (assuming that the true location of this zero is known) and the use of a full order model.

Consider the system described by

$$
y_{t}=\left(q^{-1}-3 q^{-2}\right) u_{t}+e_{t}^{o}
$$

where $\left\{e_{t}^{o}\right\}$ is Gaussian white noise of variance 0.01 . Notice that the system has exactly one NMP zero at $z_{o}=3$.

In order to initialize the algorithm, the first 20 data samples are used to estimate a second order FIR model via least squares, with a white noise input signal of variance 1. As projection mechanism, we have considered (17) with $D_{\theta_{1}}=\mathbb{R}, D_{z}=(1.001, \infty)$ and $\left[\theta_{1}^{o}, \theta_{2}^{o}\right]^{T}=[0.5,-0.6]^{T}$.

The results of a Monte Carlo simulation consisting of 100 experiments are shown in Figure 1, where the normalized (i.e., multiplied by $t$ ) variance of the estimation error of four algorithms for the estimation of the NMP zero of system (20) are shown. For this system, we notice from the figure that the RLS bootstrapped algorithm gives asymptotically the same performance as with the optimal input.

Consider now a more complex system

$$
y_{t}=\frac{(q-3)(q-0.1)}{q^{2}(q-0.5)} u_{t}+e_{t}^{o}
$$

The results of 100 experiments are shown in Figure 2. For these 100 realizations, the four algorithms have actually converged to the true NMP zero, $z_{o}=3$. From this graph it can be seen that the algorithm of Section 3 gives very good performance, even when compared with the use of the $z_{o}$-consistent input for zero estimation. The fourth method, consisting in estimating the NMP zero from a full

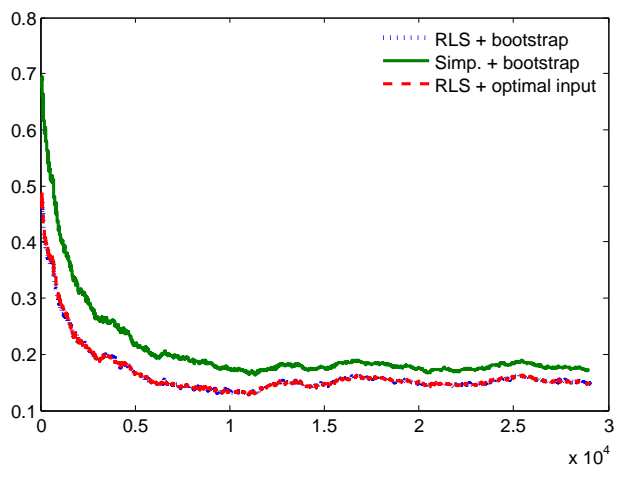

Fig. 1. Normalized variance of the estimation error for different algorithms for the estimation of NMP zeros for system (18): Recursive Least Squares (RLS) with (bootstrapped) $z_{o}$-consistent input derived from the estimated zero; the simplified algorithm of Section 3; and RLS combined with the true $z_{0}$-consistent input. As shown, the asymptotic variances of the RLS algorithms are almost indistinguishable.

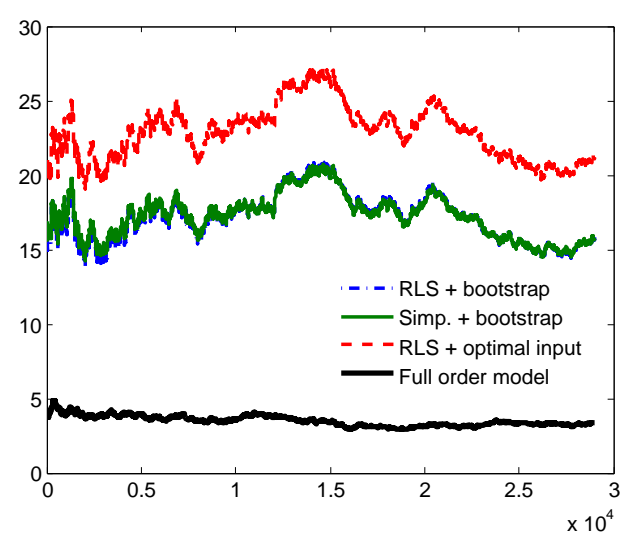

Fig. 2. Normalized variance of the estimation error for different algorithms for the estimation of NMP zeros for system (19): RLS with (bootstrapped) $z_{o}$-consistent input derived from the estimated zero; the simplified algorithm of Section 3; RLS combined with the true $z_{o}$-consistent input; and the estimated zero from a full order model obtained by recursive PEM. As shown, the asymptotic variances of RLS and the simplified algorithm with the bootstrapped input seem to be the same.

order model, gives much better performance than the other procedures. This is due to the presence of undermodelling in the model used by the other methods.

Finally, consider an even more complicated system:

$$
y_{t}=\frac{(q-3)(q-0.1)(q-0.2)(q+0.3)}{q^{4}(q-0.5)} u_{t}+\frac{q}{q-0.8} e_{t}^{o}
$$

The variance of the four algorithms is presented in Figure 3 , and a typical realization of the simplified algorithm with a bootstrapped input for this case is shown in Figure 4. As can be seen from this figure, the discrepancy in performance between using a full order model and a second order FIR model is much larger for this system than for the previous ones, this is due to the presence of heavy undermodelling.

It is important to remark that, even though in the presence of undermodelling, the proposed algorithm does not 


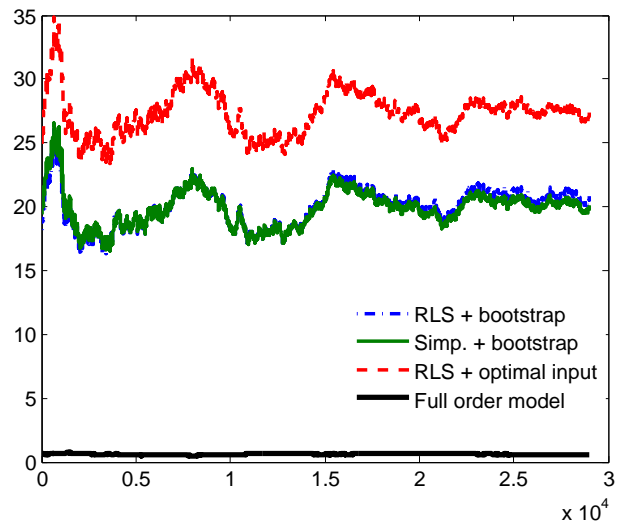

Fig. 3. Normalized variance of the estimation error for different algorithms for the estimation of NMP zeros for system (20): RLS with (bootstrapped) $z_{0}$-consistent input derived from the estimated zero; the simplified algorithm of Section 3; RLS combined with the true $z_{o}$-consistent input; and the estimated zero from a full order model obtained by recursive PEM. As shown, the asymptotic variances of RLS and the simplified algorithm with the bootstrapped input seem to be the same.

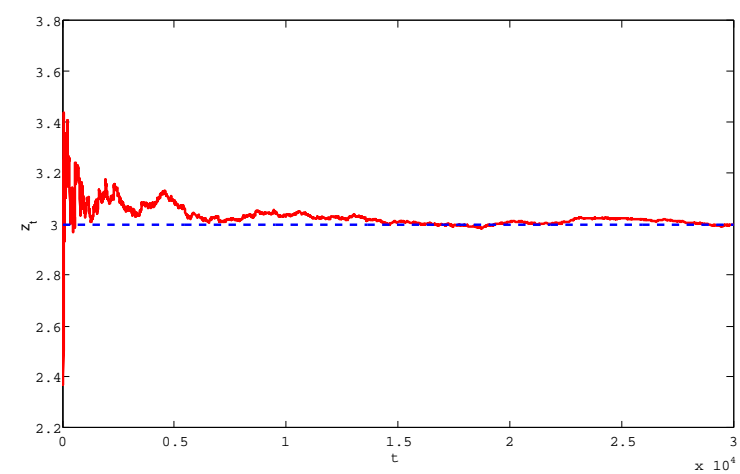

Fig. 4. Typical realization of the algorithm of Section 3 applied to system (20): solid line. True location of the zero: dashed line.

perform as well as when a full model is available, it does provide a consistent estimate. We believe this is a remarkable practical observation, since in real world applications we seldom have a model including exactly all the dynamics of the real plant, but even in this situation we can provide a consistent estimation of a relevant property of the system.

\section{CONCLUSIONS}

We have in this contribution presented a very simple algorithm for estimating a real NMP-zero of a stable rational LTI system of arbitrary order subject to white noise disturbances. The algorithm is accompanied by a convergence proof which in particular shows that if a lower bound on the magnitude of the real NMP-zero furthest away from the origin is known, then the algorithm can be initialized so that it will converge to this zero.

The convergence rate for the algorithm still has to be derived. However, it is clear from the simulation example that there is a penalty in the accuracy for the adaptive algorithm as compared with the case when a full order model is used. Since it has been shown in Gerencsér et al. [2007, 2009] that asymptotically in the sample size there is no such penalty when the true system is in the model set, the size of the performance degradation depends on the amount of undermodeling.

Finally, we remark that we were quite surprised by the ease with which it was possible to derive a convergence result for our adaptive algorithm - despite severe model/system mismatch; this especially in light of the limited results that exists under such conditions in adaptive control. It is interesting to examine the underlying reasons for this. Much of the contributions to adaptive control, especially works prior to the mid 1980s, employ the certainty equivalence principle in the control design, i.e. the latest available parameter estimate is used to compute the desired controller. Also in this contribution we employ the certainty equivalence principle, but instead of using it directly for the application (as in adaptive control) we use it when designing an experiment that is optimal from an identification perspective with respect to the end application (in our case the estimation of NMP zeros). We believe that the properties of our algorithm are very much tied to this objective.

As already pointed out in the introduction an optimal experiment has the dual objectives of enhancing system properties of interest and "hiding" system properties that are of little or no interest. We believe that both these properties facilitate the use of adaptive techniques and that these properties are what allow us to "get away" with a simple two parameter model for the problem of NMPzero estimation.

Comparing with adaptive control, the approach can be seen as the other end of the extreme: In (certainty equivalence) adaptive control one immediately tries to optimize the control objective (at the expense of the identification accuracy). Here we instead put the identification at the forefront at the cost of the application. When the application is control, dual control is the optimal balance between these two objectives, but the results we have obtained in this contribution point to that a shift towards identification as the primary objective also in adaptive control may provide valuable robustness. This is uncharted territory that awaits exploration.

\section{REFERENCES}

M. Barenthin, H. Jansson, and H. Hjalmarsson. Applications of mixed $\mathcal{H}_{\infty}$ and $\mathcal{H}_{2}$ input design in identification. In 16th World Congress on Automatic Control, Prague, Czech Republik, 2005. IFAC. Paper Tu-A13-TO/1.

D.S. Bayard, Y. Yam, and E. Mettler. A criterion for joint optimization of identification and robust control. IEEE Trans. Automatic Control, 37:986-991, 1992.

J.M. Böling and P.M. Mäkilä. On control relevant criteria in $H_{\infty}$ identification. IEEE Transactions on Automatic Control, 43(5):694-700, 1998.

R.A. de Callafon and P.M.J. Van den Hof. Connecting system identification and robust control by a factorization approach. In 11th IFAC Symposium on System Identification, volume 1, pages 117-122, Fukuoka, Japan, 1997.

S.A. Eker and M. Nikolaou. Linear control of nonlinear systems: Interplay between nonlinearity and feedback. AIChE Journal, 48(9):1957-1980, 2002.

L. Gerencsér and H. Hjalmarsson. Adaptive input design in system identification. In Proc. of the 44th IEEE Conference on Decision and Control and European Control Conference, pages 4988-4993, Seville, Spain, December 12-15 2005.

L. Gerencsér, H. Hjalmarsson, and J. Mårtensson. Adaptive input design for ARX systems. In European Control Conference, Kos, Greece, July 2-5 2007. 
L. Gerencsér, H. Hjalmarsson, and J. Mårtensson. Identification of ARX systems with non-stationary inputs asymptotic analysis with application to adaptive input design. Automatica, 45:623-633, 2009.

M. Gevers. Connecting identification and robust control: A new challenge. In Proc. IFAC/IFORS Symposium on Identification and System Parameter Estimation, Budapest, Hugary, 1991.

M. Gevers. Towards a joint design of identification and control? In H. L. Trentelman and J. C. Willems, editors, Essays on Control: Perspectives in the Theory and its Applications. Birkhäuser, 1993.

M. Gevers and L. Ljung. Optimal experiment designs with respect to the intended model application. Automatica, 22:543-554, 1986.

G.C. Goodwin. Identification and robust control: Bridging the gap. In Proceedings 7th IEEE Mediterranean Conference on Control and Automation, Haifa, Israel, 1999.

G.C. Goodwin, M. Gevers, and B. Ninness. Quantifying the error in estimated transfer functions with application to model order selection. IEEE Trans. Automatic Control, 37:913-928, 1992.

R. Hildebrand and M. Gevers. Identification for control: Optimal input design with respect to a worst-case $\nu$-gap cost function. SIAM J. Control Optim, 41(5):1586-1608, 2003.

H. Hjalmarsson. From experiment design to closed loop control. Automatica, 41(3):393-438, March 2005.

H. Hjalmarsson, J. Mårtensson, and B. Wahlberg. On some robustness issues in input design. In 14th IFAC Symposium on System Identification, Newcastle, Australia, March 2006.

E.W. Jacobsen. Identification for control of strongly interactive plants. In Proceedings AIChE Annual Meeting, San Fransico, California, USA, 1994. Paper 226ah.

H. Jansson. Experiment Design with Applications in Identification for Control. PhD thesis, S3-Automatic Control, Royal Institute of Technology, Stockholm, Sweden, 2004.

H. Jansson and H. Hjalmarsson. Mixed $\mathcal{H}_{\infty}$ and $\mathcal{H}_{2}$ input design. In IEEE Conference on Decision and Control, Bahamas, December 2004.

H. Jansson and H. Hjalmarsson. Input design via LMIs admitting frequency-wise model specifications in confidence regions. IEEE Transactions on Automatic Control, 50(10):1534-1549, 2005.

K. Lindqvist and H. Hjalmarsson. Optimal input design using linear matrix inequalities. In Proceedings of the 12th IFAC Symposium on System Identification, 2000.

K. Lindqvist and H. Hjalmarsson. Identification for control: Adaptive input design using convex optimization. In Conference on Decision and Control, pages 43264331, Orlando, Florida, USA, December 2001. IEEE.

K. Lindqvist and H. Hjalmarsson. Adaptive input design using convex optimization. Automatica, 2002. In preparation.

L. Ljung. Analysis of recursive stochastic algorithms. IEEE Trans. Automatic Control, 22:551-575, August 1977.

L. Ljung. A discussion of model accuracy in system identification. Int. Jour. of Adapt. Control and Signal Proc., 6:161-171, 1992.

L. Ljung and T. Söderström. Theory and Practice of Recursive Identification. MIT Press, Cambridge, Massachusetts, 1983.

S. Malan, M. Milanese, D. Regruto, and M. Taragna. Robust control from data via uncertainty model sets identification. In Proceedings 40th IEEE Conference on Decision and Control, Orlando, Florida, USA, December
2001. IEEE.

J. Mårtensson. Geometric Analysis of Stochastic Model Errors in System Identification. PhD thesis, Automatic Control, Electrical Engineering, Royal Institute of Technology (KTH), Stockholm, Sweden, 2007.

J. Mårtensson, H. Jansson, and H. Hjalmarsson. Input design for identification of zeros. In 16th World Congress on Automatic Control, Prague, Czech Republik, 2005. IFAC.

L. Pronzato. Optimal experimental design and some related control problems. Automatica, 44(2):303-325, February 2008.

D.E. Rivera and K.S. Jun. An integrated identification and control design methodology for multivariable process system applications. IEEE Control Systems Magazine, 20(3):25-37, 2000

C. R. Rojas. Robust Experiment Design. PhD thesis, The University of Newcastle, Newcastle, Australia, 2008.

C. R. Rojas, M. Barenthin, J. S. Welsh, and H. Hjalmarsson. The cost of complexity in identification of FIR systems. In 16th IFAC World Congress, Seoul, South Korea, 2008a.

C. R. Rojas, M. Barenthin, J. S. Welsh, and H. Hjalmarsson. The cost of complexity in system identification: The FIR case. IEEE Transactions on Automatic Control, 2008b. Submitted.

R. J. P. Schrama. Accurate models for control design: the necessity of an iterative scheme. IEEE Trans. Automatic Control, 37:991-994, 1992.

P.M.J. Van den Hof and R.J.P. Schrama. Identification and control - closed loop issues. Automatica, 31(12): 1751-1770, 1995.

B. Wahlberg and L. Ljung. Design variables for bias distribution in transfer function estimation. IEEE Trans. Automatic Control, 31:134-144, 1986.

Z. Zang, R.R. Bitmead, and M. Gevers. Iterative weighted least-squares identification and weighted LQG control design. Automatica, 31:1577-1594, 1995. 